\title{
AVALIAÇÃO DE UM JOGO SÉRIO DIGITAL DESTINADO AO PÚBLICO IDOSO UTILIZANDO O MÉTODO GAMEFLOW
}

\author{
Leo Natan Paschoal, CCHS/UNICRUZ, leonatanpaschoal@gmail.com \\ Vinícius Lopes, INF/PUCRS, vin.lopes159@gmail.com \\ Mariane Regina S. Cassenote, CCHS/UNICRUZ, mariane.sponchiado@ gmail.com \\ Cíndia R. Toniazzo Quaresma, CCHS/UNICRUZ, cquaresma@unicruz.edu.br \\ Patricia M. Mozzaquatro Chicon, CCHS/UNICRUZ, patriciamozzaquatro@gmail.com
}

\begin{abstract}
Resumo: Este artigo descreve a avaliação do jogo sério baseado em hipermídia adaptativa intitulado CLICKOLD, o qual objetiva o aprimoramento da coordenação motora fina de pessoas idosas a fim de promover a inclusão digital. Para tanto, foi utilizado o instrumento GameFlow a fim de aferir os níveis de imersão e diversão encontrados no jogo. A avaliação foi realizada com o auxílio de estudantes de graduação em Ciência da Computação que possuem familiaridade com jogos digitais. Foram considerados os seguintes critérios: Habilidades do Jogador, Concentração, Desafio, Controle, Objetivos e Feedback. A partir dos resultados obtidos foi possível inferir que grande parte dos atributos avaliados obtiveram uma classificação razoável, tendo sido encontrada uma limitação apenas no critério que se refere às habilidades do jogador. Acredita-se que esse resultado seja atribuído ao fato de o jogo não apresentar um tutorial para usuários iniciantes, além da carência de recompensas pela transposição de cada desafio.
\end{abstract}

Palavras-Chave: Avaliação de Jogo, Heurísticas em avaliação, CLICKOLD.

Abstract: This article describes an evaluation of serious game based on Adaptative Hypermedia entitled CLICKOLD, which objective the improvement of fine motor skills of elderly people in order to promote the digital social. So that, it was used the GameFlow instrument in order to check the levels of immersion and entertainment found in the game. The assessment was performed with the help of undergraduate students in computer science that have familiarity with digital games. It was considered the following criteria: Player Skills, Concentration, Challenge, Control, Objectives and Feedback. From the results obtained was possible to infer that large part of attributes assessed obtained a reasonable classification, was found a limitation just criteria that refer to the player skills. It is believed that this results to be attributed to the fact that the game not to present a tutorial for the beginner users, besides the lack of rewards by the transposition of each challenge.

Keywords: Evaluation of game, Heuristics in evaluation, CLICKOLD. 


\section{Introdução}

Jogos sérios apresentam-se como recursos promissores no contexto que abrange a aprendizagem eletrônica, pois possuem formato instrucional ou de aprendizagem. Quando utilizados como recurso didático em sala de aula, oferecem características de suporte benéficas às práticas de ensino e aprendizagem (SAVI; UBERITH, 2008). Desse modo, os jogos se inserem como artefato instrucional eficiente para personalizar as aulas tradicionais monótonas, garantindo práticas educativas atrativas e inovadoras.

Os jogos digitais têm assumido um papel importante na sociedade, caracterizando-se como recursos tecnológicos culturais. Vêm tornando-se uma atividade de lazer popular entre os mais variados tipos de público (CARVALHO; ISHITANI, 2013). Pela característica da popularidade, os jogos consomem muitas horas diárias dos jogadores, tempo este que poderia ser aproveitado para o estudo (SAVI; UBERITH, 2008). Nesse sentido, os jogos sérios, cujo objetivo principal não é o entretenimento, surgem como ferramentas fundamentais ao encontro sinergético entre aprendizado e diversão.

Em paralelo a grande popularidade dos jogos digitais, a população idosa brasileira cresce rapidamente (RIBEIRO et al., 2015). A velhice acarreta diversas mudanças na vida do indivíduo, principalmente mudanças físicas que interferem na coordenação motora, na velocidade dos movimentos, na força e na flexibilidade (MINÚSCOLI et al., 2013). Nesse contexto, Silveira (2015) salienta que a motricidade fina representa um elemento fundamental na vida do idoso, pois é ela que proporciona independência na realização de tarefas cotidianas. Ainda, a redução de força muscular pode tornar o idoso incapaz de exercer atividades rotineiras, como exemplo, utilizar o hardware mouse (SILVEIRA, 2015).

Parte da população idosa brasileira possui acesso à equipamentos eletrônicos computacionais (desktops e/ou dispositivos móveis). Sabe-se que sua utilização é capaz de melhorar habilidades manuais e cognitivas do idoso (SILVEIRA, 2015). Ainda, o computador pode proporcionar diversos recursos para o aprendizado dos idosos, visto que, conforme Mol (2011), é necessário criar estratégias para promover a aprendizagem contínua dessas pessoas. Diante desse cenário, Carvalho e Ishitani (2013) sugerem o uso de jogos sérios, visto que os mesmos fazem parte da cultura atual e refletem na inclusão social do idoso.

É notória a participação de idosos em cursos relacionados à inclusão digital, tais como oficinas de informática ou até mesmo em cursos de capacitação em softwares específicos (PREVEDELLO et al., 2015). Essa parcela da população tem perdido a resistência em utilizar recursos tecnológicos em suas atividades diárias (RIBEIRO et al., 2015). Explorar essa oportunidade, trazendo o contexto dos jogos em cursos de capacitação contínua, permitirá ao idoso aprender de maneira ativa e dinâmica, visto que os jogos sérios proporcionam o sentimento de motivação, fator responsável pelo desenvolvimento de estratégias e novas habilidades.

Considerando o contexto exposto e o apresentado na literatura científica, esta pesquisa visa demonstrar, inicialmente, um jogo sério desenvolvido para participantes de um curso de inclusão digital, assim como um experimento realizado com estudantes de graduação em Ciência da Computação. O jogo sério, denominado CLICKOLD, tem como objetivo promover um recurso para melhorar a coordenação motora fina de 
usuários idosos por intermédio do hardware mouse. Sabe-se que jogos sérios podem ser utilizados como instrumentos e são fortemente aceitos pela sociedade (TROIS, 2013). Nesse sentido, parte-se do pressuposto que sua utilização pode enriquecer as aulas de inclusão digital.

Visando evidenciar a qualidade e mensurar o prazer do usuário com o jogo, o mesmo foi proposto a uma turma de estudantes a fim de realizar um teste prévio, antes de o jogo ser apresentado ao público idoso do curso de inclusão digital. Para a avaliação foi utilizado o método GameFlow, o qual se destina à avaliação de jogos utilizando critérios que identificam o potencial oferecido ao usuário. Salienta-se que se optou pelo público jovem para realização de testes iniciais, pois os mesmos estão familiarizados com jogos e podem avaliar alguns critérios de mecânica do jogo que normalmente não são selecionados durante a avaliação de usabilidade se realizada diretamente com o público idoso. É condizente informar que a usabilidade é um critério que será investigado futuramente, por meio da aplicação do jogo diretamente no público alvo, pois é por meio desse quesito que é possível identificar se o jogador sente prazer em jogar (CARVALHO; ISHITANI, 2013).

Essa pesquisa está estruturada da seguinte forma: a segunda seção contempla uma contextualização sobre a informática na velhice; a terceira seção apresenta um breve referencial teórico a cerca dos jogos sérios e também elucida o jogo CLICKOLD; na quarta seção é descrito o método GameFlow e suas heurísticas; os métodos de pesquisa utilizados neste estudo são expostos na quinta seção; a sexta seção apresenta os resultados decorrentes do teste realizado; por fim, a sétima seção apresenta as considerações finais desse estudo, seguido das referências bibliográficas.

\section{Informática no envelhecimento humano}

A população idosa brasileira tem crescido vertiginosamente, constituindo grupos de pessoas que buscam manterem-se ativos e produtivos por meio de atividades significativas e desafiantes. Dentre estas, o uso do computador e outros artefatos tecnológicos têm sido fatores relevantes, tanto na inclusão social e digital do idoso, quanto na manutenção das suas habilidades motoras e cognitivas.

É importante destacar que não se trata apenas reproduzir a informática, mas integrá-la ao cotidiano, tornando-a não apenas técnica, mas cada vez mais próxima das necessidades e vivências dos idosos, seja na forma de entretenimento, comunicação, trabalho ou desempenho de atividades diárias com autonomia. Conforme Prevedello et al. (2015, p. 3) com a facilidade de acesso às operações tecnológicas aos idosos, "tornase possível que os mesmos desempenhem tarefas cotidianas de forma mais segura, rápida e confiável, dando-lhes uma liberdade que outrora não existia".

Aspectos relevantes no contexto do envelhecimento são as mudanças fisiológicas e nas capacidades perceptivas e sensoriais. Destaca-se a redução de habilidades motoras como a coordenação motora fina e viso-motora. De acordo com Piret, S. e Béziers (1992) a coordenação motora fina relaciona-se com a harmonia e precisão dos movimentos finos das mãos, pés e rosto, ou coordenação dos músculos pequenos para atividades finas, e a viso-motora é a coordenação dos movimentos orientada pela visão, associada a outras habilidades: olho-mão, olho-pé.

Assim, a inserção da informática por meio de experiências com jogos computacionais, por exemplo, em que se estimulam tais especificidades da coordenação e percepção, acaba por contribuir para o aumento da qualidade de vida do idoso. Por 
conseguinte, influencia-se diretamente no desempenho quanto ao domínio do computador, bem como a manutenção das capacidades motoras e cognitivas nesta fase da vida. Segundo Alves et al. (2007) a informática na vida do idoso é um fator de inclusão, socialização e a valorização dos conhecimentos do indivíduo, permitindo ao mesmo estar conectado, um dos seus interesses, conforme aponta a pesquisa do Comitê Gestor da Internet ${ }^{1}$.

\section{Jogos Sérios}

Os jogos sérios digitais são mídias que têm o potencial de motivar e fazer com que o usuário desenvolva habilidades e estratégias (SAVI; UBERITH, 2008). Esses recursos são vastamente utilizados por instituições de ensino a fim de auxiliar estudantes na interpretação e fixação de conceitos, assim como no reforço de conteúdos. O seu objetivo principal não é o entretenimento, mas sim servir para um propósito sério, tal como a educação (KRASSMANN et al., 2015).

Utilizar jogos para disseminar conhecimento é uma estratégia adotada por alguns pesquisadores como Silva (2012), Trois (2013) e Krassmann et al. (2015). Os jogos conseguem proporcionar uma realidade na qual é possível aprender de maneira divertida e inspiradora, visto que fazem com que o usuário se envolva em um mundo virtual com situações que em alguns casos seriam difíceis de serem resolvidas. É comum o seu uso em diversos contextos educativos, como na área da saúde (SILVA, 2012). Existem jogos que permitem a realização de simulações de atividades médicas práticas que não causam riscos aos seres humanos. Nesse sentido, o estudante jogador aprenderá o conteúdo de maneira mais profunda.

A ideologia da usabilidade de um jogo sério consiste em utilizar a sua essência a fim de fazer com que o estudante aprenda o conteúdo de uma disciplina (SILVA, 2012). Desse modo, o ensino baseado em jogos parte da premissa da minimização do fracasso (MATTAR, 2010), visto que o estudante poderá errar e repetir suas ações sempre que necessitar, sem grandes prejuízos, o que justifica o seu uso em diferentes contextos.

Alguns benefícios dos jogos sérios são destacados por Savi e Uberith (2008): efeito de motivação, desenvolvimento de habilidades cognitivas, aprendizagem por descoberta. Esses benefícios correspondem à característica do ambiente de capturar a atenção do estudante por intermédio de recursos gráficos, narrativos e sonoros. Por esse motivo, inserem-se como uma alternativa às atividades repetitivas normalmente solicitadas em aulas tradicionais.

Entre os diversos benefícios dos jogos digitais, salienta-se o fato de eles permitirem o desenvolvimento de coordenação motora fina por meio do hardware mouse (SAVI; UBERITH, 2008). Diante disso, evidencia-se a sua aplicabilidade com o público idoso. A próxima seção tem como objetivo apresentar o jogo sério digital CLICKOLD.

\subsection{CLICKOLD}

Baseado no estilo de jogo point-and-click em que a interação com o mesmo ocorria quase que inteiramente por meio do hardware mouse (TORRENTE, 2010), foi

\footnotetext{
${ }^{1}$ http://www.cgi.br/
} 
desenvolvido em 2015 o CLICKOLD, cujo nome representa a união em inglês das palavras "clique" e "idoso". O jogo instiga a interação do usuário com o cenário proposto por meio de ações pré-definidas associadas à hipermídia adaptativa (LOPES, 2015).

O enredo do jogo consiste em um personagem idoso, em que o gênero é escolhido pelo usuário, realizando tarefas do dia a dia em sua casa para se preparar para a visita de seu neto. O jogo possui três fases, onde cada uma representa um cômodo de uma casa, sendo eles: quarto, banheiro e cozinha. Na Figura 1 pode ser vista uma tela do jogo, representado a sua primeira fase.

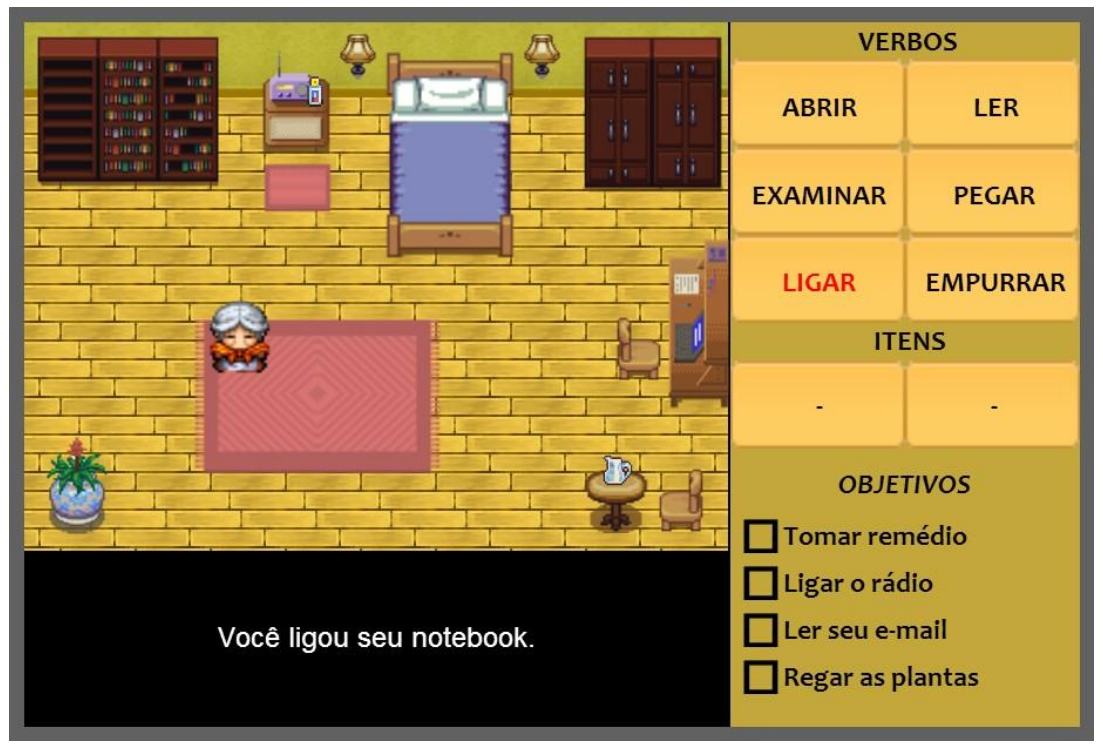

Figura 1 - Primeira fase do CLICKOLD: o quarto

Para concluir o desafio, o usuário deve cumprir todos os objetivos propostos no canto inferior direito da tela. Para isso, o mesmo deverá interagir com o cenário de duas formas, por interação direta com os objetos ou utilizando verbos pré-selecionados para especificar a interação com os mesmos.

Existem duas versões de cada fase, na primeira delas, ilustrada na Figura 1, a interação ocorre com o usuário selecionando um dos botões da lateral direita do jogo e em seguida clicando no cenário para que a ação selecionada naquele botão seja efetuada. Com a técnica de hipermídia adaptativa chamada anotação adaptativa, o botão selecionado muda a cor da sua escrita de preto para vermelho, destacando-o para facilitar visualmente o entendimento do usuário.

$\mathrm{Na}$ segunda versão da fase, ilustrada na Figura 2, a interação ocorre de forma direta com o cenário, sem a necessidade de cliques para selecionar os botões com ações. Assim, ao clicar no objeto selecionado é executada a melhor ação possível pare ele, sendo necessários muito menos cliques e executando a tarefa em menos tempo do que na outra versão. Foi utilizada a técnica de ocultação, também pertencente a hipermídia adaptativa, para desabilitar os botões tanto graficamente quanto efetivamente na tela do jogo.

O modo como o próprio usuário interage no jogo é o que define qual versão do cenário o mesmo obterá. Ao final de cada fase é contabilizado o número de cliques dados e o tempo em segundos levado para a conclusão de todos os objetivos, caso esses valores fiquem abaixo da média estabelecida pelo jogo, a próxima fase será a versão 
com o uso de botões, caso contrário a próxima fase será a versão onde os botões são desabilitados.

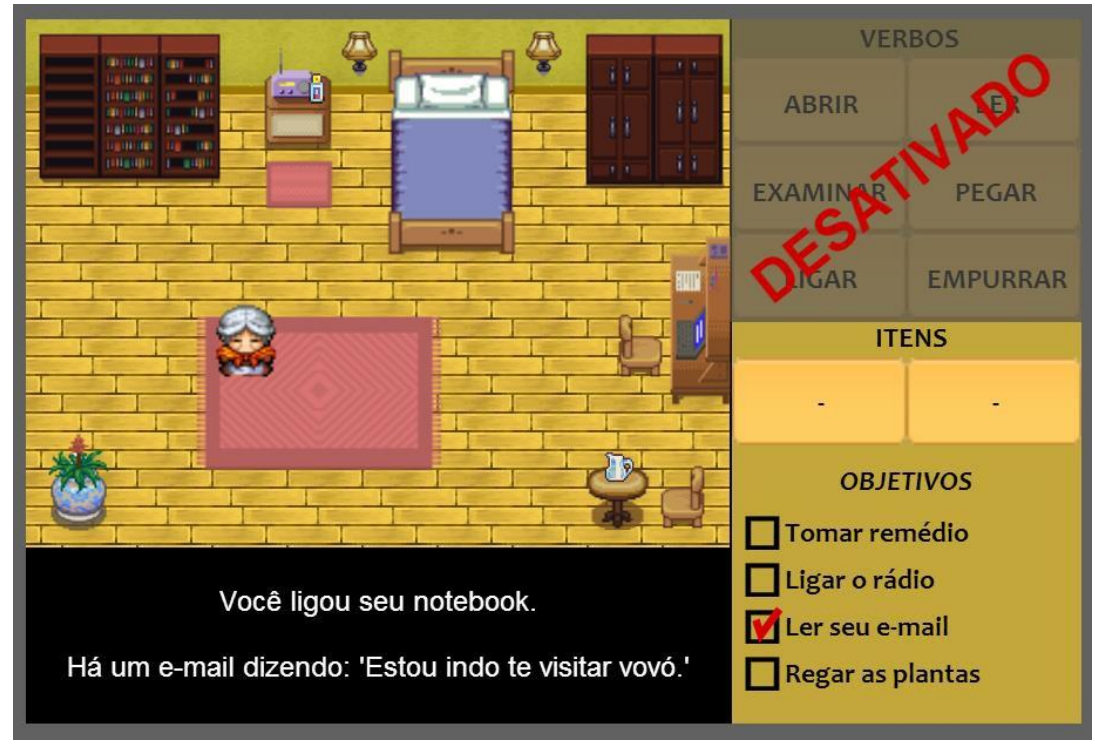

Figure 2 - Primeira fase do CLICKOLD em sua versão alternativa

Esse processo se repete até o final do jogo, a fim de se adaptar a cada usuário, seu estilo de aprendizado, seu nível de conhecimento e suas capacidades cognitivas e motoras finas. Portanto, objetivando-se que o mesmo aprenda a utilizar não somente os recursos do jogo de forma efetiva, mas também os recursos de um computador em si, constituindo-se em uma ferramenta de ensino eficaz para a inclusão digital de idosos.

\section{GameFlow}

O GameFlow, proposto por Sweetser e Wyeth (2005), é um modelo para projeto, avaliação e compreensão da diversão em jogos. De acordo com Breyer (2008), o diferencial desse recurso é ser centrado na integração, além de se tratar de um método que avalia a diversão dos usuários e não a usabilidade em si, como a maior parte dos modelos existentes.

Para a elaboração e validação dessa ferramenta, foram aplicadas heurísticas relacionadas à usabilidade e à experiência do usuário. A Teoria de Fluxo (Flow Theory), desenvolvida por Csikszentmihalyi (1990), apresentada como principal fundamentação teórica para definição do GameFlow, afirma que um indivíduo se encontra em estado de flow quando imerso, concentrado e envolvido em uma atividade. $\mathrm{O}$ modelo consiste em oito elementos centrais cujos conceitos incluem um número variável de critérios que estão relacionados aos elementos da Teoria de Fluxo e que definem a presença ou não de diversão nos jogos.

O modelo é subdividido entre os elementos concentração, desafio, habilidade do jogador, controle, objetivos claros, feedback, imersão e integração social, os quais serão detalhados a seguir, conforme o entendimento de Neves et al.(2014):

Tabela 1 - Descrição dos critérios de avaliação

\begin{tabular}{|l|l|}
\hline \multirow{2}{*}{ Concentração } & $\begin{array}{l}\text { O nível de interesse no jogo é proporcional à concentração exigida por uma dada atividade. É } \\
\text { necessário manter uma carga de trabalho adequada às habilidades do jogador, considerando seus } \\
\text { limites cognitivos e de memória. Devem ser evitadas distrações não relacionadas à tarefa } \\
\text { principal a ser realizada. }\end{array}$ \\
\hline
\end{tabular}




\begin{tabular}{|c|c|}
\hline Desafio & $\begin{array}{l}\text { Trata-se de um dos aspectos mais importantes de um jogo, uma vez que desafios maiores que as } \\
\text { habilidades do jogador podem gerar ansiedade, enquanto desafios menores podem causar apatia e } \\
\text { indiferença. Um ritmo apropriado no jogo possibilita pressão, mas não frustra o usuário. }\end{array}$ \\
\hline $\begin{array}{l}\text { Habilidades do } \\
\text { jogador }\end{array}$ & $\begin{array}{l}\text { As habilidades do usuário devem ser desenvolvidas pelo próprio jogo para que se tenha um } \\
\text { equilíbrio com os desafios apresentados. Assim, o processo de jogar se torna prazeroso, divertido } \\
\text { e desafiador. Outro aspecto relevante consiste na interface e nos dispositivos de entrada } \\
\text { utilizados. Ambos devem ser fáceis de compreender e manusear, seguindo os padrões da } \\
\text { indústria e utilizando analogias com o mundo real. }\end{array}$ \\
\hline Controle & $\begin{array}{l}\text { O usuário deve ter controle efetivo sobre elementos do universo do jogo, personagens e objetos, } \\
\text { além de os elementos da interface de usuário, informações relevantes para seus objetivos e } \\
\text { dispositivos de entrada. Ademais, são importantes os aspectos que se referem ao controle sobre o } \\
\text { andamento do jogo, realizado por meio das possibilidades de interromper e retomar, salvar o } \\
\text { estado do jogo e retornar a um estado anteriormente salvo. }\end{array}$ \\
\hline $\begin{array}{l}\text { Objetivos } \\
\text { claros }\end{array}$ & $\begin{array}{l}\text { Os objetivos do jogo devem ser compreendidos de forma clara e concisa. Há os objetivos } \\
\text { principais, como a história ou contexto geral do jogo, objetivos relacionados a cada nível ou fase } \\
\text { e os objetivos correspondentes a cada tarefa a ser cumprida. }\end{array}$ \\
\hline Feedback & $\begin{array}{l}\text { É extremamente importante que o jogador receba feedbacks sobre suas ações e evolução no jogo } \\
\text { a todo instante e no momento em que desejar. Sua pontuação e status no jogo devem estar } \\
\text { acessíveis, a fim de auxiliá-lo a mensurar seu progresso em direção a um objetivo, a compreender } \\
\text { o que errou e como corrigir esse erro. }\end{array}$ \\
\hline Imersão & $\begin{array}{l}\text { Jogos devem transportar o usuário para um nível de envolvimento emocional e visceral, levando- } \\
\text { os a esquecer que estão jogando. Efeitos sonoros e visuais são bons recursos nesse sentido, assim } \\
\text { como o enredo e o plano de fundo, levando os jogadores a se sentirem como parte da história. }\end{array}$ \\
\hline Interação social & $\begin{array}{l}\text { Este não é um elemento do flow, mas sim um recurso que exerce grande atração sobre o usuário. } \\
\text { Assim, jogos devem criar e suportar oportunidades que possibilitem interação social, como } \\
\text { competição, cooperação e conexão entre os jogadores, além de chats e salas online. }\end{array}$ \\
\hline
\end{tabular}

\section{Procedimentos metodológicos}

Com o propósito de esclarecer e identificar os pontos positivos do jogo e também as suas fragilidades, essa pesquisa foi executada em três etapas:

A primeira etapa consistiu no estudo teórico acerca do assunto de jogos sérios, informática na vida do idoso e o método de avaliação GameFlow. Desse modo, foi possível encontrar subsídios para a fundamentação bibliográfica que alicerça o trabalho.

Após o aprofundamento realizado no contexto teórico, na segunda etapa dessa pesquisa foi realizada a instalação das ferramentas necessárias para suportar a execução do jogo sério CLICKOLD. Conforme Lopes (2015), o jogo pode ser executado em diferentes plataformas por intermédio de um browser. Nesse sentido, o jogo foi instalado em servidor, com auxílio do software Xampp, o qual apresenta os seguintes recursos: sistema gerenciador de banco de dados MySQL - versão 5.6.16; Apache para hospedagem do sistema; além de oferece suporte a interpretação da linguagem de programação Hypertext Preprocessor - versão 5.5.11. Posteriormente, o endereço público de acesso ao jogo foi compartilhado para atender as especificidades da terceira etapa.

Considerando os objetivos supracitados, assim como a avaliação da satisfação que um jogador possui ao jogar e evidenciar se o jogo promove diversão aos usuários, na terceira etapa realizou-se a aplicação de um questionário eletrônico. O questionário foi composto por 32 questões, sendo que 28 são oriundas do método GameFlow e 4 refere-se a dados demográficos de cada participante. O questionário foi construído com auxílio da ferramenta Google Drive, disponível em: 〈http://goo.gl/forms/uEjUieifZr〉.

A avaliação do jogo foi efetivada com cinco alunos do curso de graduação em Ciência da Computação de uma Instituição de Ensino Superior Comunitária. Neves et al. (2014) validou sua pesquisa com três pessoas e acreditou ser esse um número satisfatório de participantes. Optou-se por utilizar esse púbico, pois os estudantes estão 
familiarizados com o uso do computador e jogos digitais. Neste sentido, é possível mensurar a diversão e o prazer que o jogo proporciona, além de identificar possíveis melhorias.

Optou-se por retirar os critérios de imersão e interação do GameFlow, pois o CLICKOLD não apresenta recursos que possibilitam um envolvimento do jogador com a história do jogo, visto que o mesmo é voltado ao público idoso, não sendo classificado como multiplayer. Logo, restaram-se seis critérios a serem utilizados.

\section{Resultados do experimento}

As quatro primeiras questões buscaram identificar caraterísticas demográficas dos participantes. Constatou-se que $100 \%$ dos jogadores possuíam o sexo masculino, e todos eles possuem conhecimentos em algoritmos e programação, pois cursaram disciplinas relacionadas à essas competências na graduação. Aos serem questionados quanto a frequência que jogam, apontou-se que $60 \%$ dos participantes possuem hábito de jogar diariamente ou semanalmente. A Tabela 2 mostra dados da faixa etária e a frequência que os participantes da pesquisa costumam jogar.

Tabela 2 - Dados demográficos dos jogadores

\begin{tabular}{|c|c|c|c|c|c|c|c|}
\hline & \multicolumn{3}{|c|}{ Idade } & \multicolumn{4}{c|}{ Frequência que joga } \\
\hline & $<=17$ & 18 ou 19 & $>=20$ & Diariamente & Semanalmente & Mensalmente & Não tem hábito de jogar \\
\hline Indiv & 2 & 1 & 2 & 2 & 1 & 0 & 2 \\
\hline$(\%)$ & $(40)$ & $(20)$ & $(40)$ & $(40)$ & $(20)$ & $(0)$ & $(40)$ \\
\hline
\end{tabular}

Os participantes possuíam cinco opções de respostas para cada questão originada do método GameFlow. Essas opções são descritas a seguir: não se aplica; deveria ter, mas não tem; ruim; médio; bom. Desse modo, cada opção de resposta equivale a uma nota que pode variar em um intervalo de 0 (não se aplica) a 4 (bom).

Após a tabulação das respostas, foi realizada uma média por critério de avaliação. Constatou-se que o critério que obteve maior limitação, ou seja, obteve uma pontuação média inferior a três pontos, foi Habilidades do jogador, conforme pode ser observado na quarta coluna da segunda linha da Tabela 3. Acredita-se que os principais pontos considerados pelos avaliadores ao analisar os critérios de Habilidade do jogador, foram o fato de o jogo não apresenta um tutorial e também a ausência de recompensas pelo cumprimento de cada desafio. Os demais critérios obtiveram uma pontuação maior ou igual a três, garantindo uma classificação média.

O critério de Concentração obteve o melhor resultado, evidenciando que o jogador não é sobrecarregado e que a carga de trabalho é adequada aos limites de cognição, percepção e memória do usuário. O critério Desafio obteve um resultado significativo no sentido de que as fases apresentadas são adequadas às habilidades do jogador. No critério Controle, a pergunta que obteve a maior pontuação evidencia que o usuário se sente no controle da interface. No critério Objetivos, é destacado pelos avaliadores que as principais metas do jogo são facilmente identificadas. Por fim, o critério Feedback apontou que é o jogo poderia emitir mais retorno ao jogador.

Os autores Vahldick et al. (2015), também utilizaram o método GameFlow em sua pesquisa. Os mesmos transformaram cada média obtida por critério numa proporção entre 0 a 100. Nessa pesquisa, foi realizado este procedimento, o que apontou que todos os critérios apresentaram médias superiores a 60 .

Tabela 3. Média dos critérios GameFlow 


\begin{tabular}{|c|c|c|c|c|c|c|}
\hline & Concentração & Desafio & $\begin{array}{c}\text { Habilidades } \\
\text { do jogador }\end{array}$ & Controle & Objetivos & Feedback \\
\hline $0-4$ & 3,5 & 3,2 & 2,6 & 3,2 & 3,2 & 3,0 \\
\hline $0-100$ & $(87,5)$ & $(80)$ & $(65)$ & $(80)$ & $(80)$ & $(75)$ \\
\hline
\end{tabular}

Foi calculada a medida descritiva de posição média obtida pelos seis critérios utilizados para avaliar o jogo CLICKOLD. Como resultado obteve-se um valor total de 3,1 pontos. Neste sentido, foi possível identificar falhas no jogo, principalmente no quesito Habilidades do jogador. Em geral, o jogo obteve uma avaliação mediana, pois houve uma pontuação satisfatória no critério Concentração, também evidenciou uma baixa pontuação no critério Habilidades do jogador.

\section{Considerações finais}

O objetivo desse artigo foi aferir os níveis de imersão e diversão encontrados no jogo CLICKOLD. Para tanto, foi utilizado o método de avaliação GameFlow. A proposta inicial foi considerar todos os sete elementos de avaliação do método, entretanto, pelas características que o jogo possui, os critérios de imersão e interação foram desconsiderados. Logo, visou-se detectar a qualidade do jogo e identificar se o jogador sente prazer ao jogar. Por estar recém-implementado, optou-se por avaliá-lo com pessoas que apresentam familiaridade com o uso de computador e jogos eletrônicos. Por esse motivo, o jogo foi avaliado por estudantes de Ciência da Computação. Desse modo, neste artigo não foi investigado se o jogo é um recurso eficiente para melhorar a coordenação motora fina de idosos, atributo que será avaliado futuramente.

Com a aplicação do método foi possível identificar algumas potencialidades e fragilidades do jogo, conforme foi descrito na sexta seção desse artigo. Portanto, algumas modificações deverão ser realizadas a fim de melhorar aspectos de jogabilidade da ferramenta, tais como: acrescentar novos feedbacks, tutoriais, opções de ajuda/dicas.

É condizente destacar que a análise e o experimento realizado possuem algumas limitações. O número de participantes, por exemplo, foi limitado a cinco estudantes do curso de Ciência da Computação. Todos os avaliadores foram voluntários e nenhum deles faz parte do grupo investigador deste trabalho. Seria interessante utilizar um número mais expressivo de participantes, pois assim seria possível realizar uma análise estatística mais precisa, assim como identificar percentis e desvio padrão, visto que com o tamanho da amostra utilizada ela não possui valores significativos.

Futuramente, almeja-se a aplicação do jogo à idosos e realização de testes por meio do método de avaliação System Usability Scale (SUS), pois o mesmo permitirá avaliar a usabilidade do jogo. Desse modo, será possível identificar a usabilidade do jogo com os participantes do curso de inclusão digital.

\section{Referências Bibliográficas}

KREIS, R. A.; ALVES, V. P.; CÁRDENAS, C. J.; KARNIKOWSKI, M. G. O. O impacto da informática na vida do idoso. Kairós, São Paulo, 10(2), dez. 2007, pp. 153-168.

BREYER, F. B. Avaliação heurística para protótipos de jogos digitais: adaptação do método de heurísticas para a aplicação no primeiro protótipo funcional de jogos digitais. Centro de Artes e Comunicação/Universidade Federal de Pernambuco. 2008, 103p. Dissertação de Mestrado.

CARVALHO, R. N. S.; ISHITANI, L. Fatores motivacionais para desenvolvimento de mobile serious games com foco no público da terceira idade: uma revisão de literatura. Educação Temática Digital (ETD), Campinas, SP, v. 15, n. 1, p.16-32, 2013. 
CSIKSZENTMIHALYI, M. Flow: the psychology of optimal experience. Harper \& Row. New York: [s.n.], 1990.

KRASSMANN, A. L.; PASCHOAL, L. N.; FALCADE, A.; MEDINA, R. D. JASPION: Jogo Sério Educacional Sensível ao Contexto Integrado ao Mundo Virtual OpenSim. In: XXVI Simpósio Brasileiro de Informática na Educação, Maceió. p. 429-438.

LOPES, V. CLICKOLD: software interativo orientado ao idoso como processo de inclusão digital. Centro de Ciências Humanas e Sociais /Universidade de Cruz Alta, 2015. Trabalho de Conclusão de Curso.

MATTAR, J. (2010). Games em educação: como nativos digitais aprendem. São Paulo: Person, 2010.

MINÚSCOLI, A. V. F. et al. Síndrome da Fragilidade: elucidando conceitos e propostas de avaliação e tratamento. In: Dias, C. P.; Guimarães, J. C. F. (Org.). Desvendando o envelhecimento. Caxias do Sul: Faculdade da Serra Gaúcha, p. 45-55, 2013.

MOL, M.A. (2011). Recomendações de usabilidade para interface de aplicativos para smartphones com foco na terceira idade. Dissertação (Mestrado em Informática), Pontifícia Universidade Católica de Minas Gerais.

NEVES, D. E.; SANTOS, L. G. N. O.; SANTANA, R. C.; ISHITANI, L.. Avaliação de jogos sérios casuais usando o método GameFlow. Revista Brasileira de Computação Aplicada (RBCA), Passo Fundo, v. 6, n. 1, p. 45-59, abr. 2014.

PIRET, S.; BÉZIERS, M. M. A Coordenação Motora. São Paulo: Summus, 1992.

PREVEDELlO, J. D. G.; TECHIO, G. B.; QUARESMA, C. R. T.; CASSENOTE, M. R. S.; CHICON, P. M. M.; ANTONIAZZI, R.L. Inclusão digital do idoso: contribuições das redes sociais. In: XVIII Seminário Internacional de Educação no Mercosul, 2015.

RIBEIRO, S. C.; MATTEDI, A. P.; SEABRA, R. D. Avaliação a usabilidade de websites com ênfase em usuários idosos: um estudo de caso. Revista Novas Tecnologias na Educação (RENOTE), v.13, n.2, dez, 2015.

SAVI, R.; ULBRICHT, V. R. Jogos digitais educacionais: benefícios e desafios. Revista Novas Tecnologias na Educação (RENOTE), Porto Alegre, RS, v.6, n.2, 2008.

SILVA, T. G. Jogos Sérios em Mundos Virtuais: Uma abordagem para o ensinoaprendizagem de teste de software. Centro de Tecnologia/Universidade Federal de Santa Maria, 2012, 88p. Dissertação de Mestrado.

SILVEIRA, M. M. Desempenho cognitivo, estado emocional, qualidade de vida e habilidade motora manual de idosos participantes de oficinas de informática. Instituto de Geriatria e Gerontologia/ Pontifícia Universidade Católica do Rio Grande do Sul, 2015, 160p. Tese de Doutorado.

SWEETSER, P.; WYETH. P. GameFlow: A Model for Evaluating Player Enjoyment in Games. The University of Wueensland, St Lucia, Australia. 2005.

TORRENTE, J. BLANCO, Á DEL; MARCHIORI, E. J.; MORENO-GER P.; FERNÁNDEZMANJÓN, B. <e-Adventure>: Introducing educational games in the learning process. In: IEEE EDUCON 2010 Conference, Madrid, 2010, pp. 1121-1126.

TROIS, S. Propostas de Heurísticas para Adaptação de Conteúdo Instrucional a Games Educativos. Programa de Pós-Graduação em Design /Universidade Federal do Rio Grande do Sul, 2013, 135p. Dissertação de Mestrado.

VAHLDICK, A.; MENDES, A. J.; MARCELINO, M. J.; HOGENN, M.; SCHOEFFEL, P.. Testando a Diversão em um Jogo Sério para o Aprendizado Introdutório de Programação. In: XXIII Workshop sobre Educação em Computação, 2015. 